\title{
Classification of Gram-negative bacilli according to their resistance to antimicrobials in an infectious disease clinic
}

\author{
Lucian Giubelan \\ Infectious Diseases Clinic, University of Medicine and Pharmacy, Craiova, Romania
}

\begin{abstract}
Objectives. Classification on multiple criteria of Gram-negative bacilli (GNBs) according to antibiotic resistance.

Material and method. Retrospective study (January 2017-December 2018) carried out in the Infectious Diseases Clinic from Craiova; GNBs were identified using the Vitek 2 automated system, which subsequently established their sensitivity to antimicrobials; GNBs were classified based on an arbitrary score from 1 (minimum) to 5 (maximum) based on the multiple antibiotic resistance index (MAR), the percentage of multidrug resistant strains (MDR) and the percentage of extended resistance strains (XDR). The final classification represents the sum of the points awarded for each category considered.

Results. The following GNBs were considered: Escherichia coli $(n=720)$, Klebsiella pneumoniae $(n=335)$, Pseudomonas aeruginosa $(n=139)$, Proteus mirabilis $(n=60)$ and Acinetobacter baumannii $(n=29)$. MAR values are: Acinetobacter baumannii $(\mathrm{Ab})-0.6$, Proteus mirabilis $(\mathrm{Pm})-0.52$, Pseudomonas aeruginosa $(\mathrm{Pa})-0.51$, Klebsiella pneumoniae $(\mathrm{Kp})-0.37$ and Escherichia coli $(\mathrm{Ec})-0.23$. The percentage of MDR strains is: $\mathrm{Pm}-76.67 \%, \mathrm{Kp}-68.86 \%, \mathrm{~Pa}-58.71 \%$, Ec $-51.94 \%$ and $\mathrm{Ab}-51.72 \%$; XDR strains were identified for $\mathrm{Ab}-17.24 \%$ and $\mathrm{Pa}-6.47 \%$. The final classification of GNBs is as follows: $\mathrm{Pa}-12 \mathrm{p}, \mathrm{Ab}-11$ $p, P m-7 p, K p-6 p, E c-3 p$.

Conclusions. Depending on the resistance profile on multiple criteria, the classification of the studied Gram-negative bacteria is as follows: $\mathrm{Pa}, \mathrm{Ab}, \mathrm{Pm}, \mathrm{Kp}, \mathrm{Ec}$.
\end{abstract}

Keywords: Gram-negative bacilli, MAR, MDR, XDR

\section{BACKGROUND}

Gram-negative bacilli are an important category of microorganisms, responsible for human infections located in various sites such as the meningeal space, lungs, urinary tract, intra-abdominal space, bones, joints or wounds. Along with Gram-positive bacteria (particularly Staphylococcus spp.) they are the most important category of microorganisms responsible for sepsis / septicemia and are also involved in healthcare associated infections [1-3].

Antimicrobial resistance is considered one of the greatest threats to humanity (alongside the climate changes), it is worldwide spreaded (with varying degrees) and it was recognozed as a major health prob- lem by the United Nations in a special meeting (September 2016) [4]. In 2017 the World Health Organisation (WHO) has published a list of pathogens for which new antibiotics are urgently needed due to their resistance to antibiotics [5]. On that list 5 out of 12 pathogens are Gram-negative bacilli and, more important, the first category (Critical) contains only this kind of germs (carbapenems resistant Acinetobacter baumannii, carbapenems resistant Pseudomonas aeruginosa, Enterobacteriaceae resistant to carbapenems or producing extensive spectrum betalactamases). More over, in 2015 plasmidmediated polymyxin-resistance gene were discovered in China (polymyxins being considered "the last re- 
sort” for the treatment of infections due to Gram-negative bacilli).

\section{OBJECTIVE}

The study aims to classify the Gram-negative bacilli (GNBs) isolated in an infectious disease clinic according to their resistance to antimicrobials, using multiple separation criteria.

\section{MATERIAL AND METHODS}

A retrospective study (January 2017 - December 2018) was conducted in the Infectious Disease Clinic from Craiova. The GNBs were identified using the Vitek 2 automated system, which subsequently established their susceptibility to antimicrobials. GNBs were classified based on an arbitrary score from 1 (minimum, given to the last ranked) to 5 (maximum, given to the first ranked), depending on the multiple index of antibiotic resistance (MAR), the percentage of multidrug resistant strains (MDR) and extended drug resistant stains (XDR). The final classification represents the sum of the points awarded for each category considered. The calculation of the MAR and the interpretation of results are based on the work of Krumpelman PH [7], while the criteria for defining MDR and, respectively, XDR for the GNBs are those proposed by Magiorakos et al [8]. Most strains were tested to 17 antibiotics and, in the presence of resistance to most of them, the testing was extended including another 9 antimicrobials. Commonly used drugs were: Amikacin, Ampicillin, Ampicillin-Sulbactam, Cefazolin, Cefepime, Cefoxitin, Ceftazidime, Ceftriaxone, Ciprofloxacin, Ertapenem, Gentamicin, Levofloxacin, Meropenem, Nitrofurantoin, Piperacillin, Tobramycin, Trimethoprim-Sulfamethoxazole. Extensive testing included: Aztreonam, Imipenem-Cilastatin, Minocycline, Pefloxacin, Piperacillin-Tazobactam, Polymixyn E, Ticarcillin, Ticarcillin-Clavulanic acid; Rifampicin was also introduced for the Acinetobacter baumannii strains.

\section{RESULTS}

Demographic data - The median age of patients was 60 years (limits: $<1$ year -94 years). Most patients were adults (1197, 88.14\%), female (701, $51.52 \%)$ and from urban areas (730, 53.75\%).

We have identified 1358 strains of GNBs (see details in Table 1). For the purpose of the study we have selected only the strains of Escherichia coli (Ec), Klebsiella pneumoniae (Kp), Pseudomonas aeruginosa $(\mathrm{Pa})$, Proteus mirabilis $(\mathrm{Pm})$, and Acinetobacter baumannii (Ab), which represent 1283 isolates (94.47\% of the total).

TABLE 1. GNBs species and their count

\begin{tabular}{|c|c|}
\hline GNBs & Number of isolates \\
\hline Escherichia coli & 720 \\
\hline Klebsiella pneumoniae & 335 \\
\hline Pseudomonas aeruginosa & 139 \\
\hline Proteus mirabilis & 60 \\
\hline Acinetobacter baumannii & 29 \\
\hline Serratia marcescens & 11 \\
\hline Klebsiella oxytoca & 9 \\
\hline Enterobacter cloacae complex & 7 \\
\hline Citrobacter freundii & 8 \\
\hline Raoultella planticola & 5 \\
\hline Raoultella ornithinolytica & 4 \\
\hline Salmonella enterica & 4 \\
\hline Sphingomonas paucimobilis & 4 \\
\hline Morganella morganii & 4 \\
\hline Burkholderia cepacia & 3 \\
\hline Enterobacter aerogenes & 3 \\
\hline Bordetella bronchiseptica & 2 \\
\hline Stenotrophomonas maltophila & 2 \\
\hline Pantoea dispersa & 2 \\
\hline Pseudomonas putida & 2 \\
\hline Shigella sonnei & 1 \\
\hline Leclercia adecarboxylata & 1 \\
\hline Serratia plymuthica & 1 \\
\hline Cronobacter sakazakii & 1 \\
\hline Pantoea agglomerans & 1 \\
\hline
\end{tabular}

The pathological products from which the GNBs were isolated are presented in Table 2.

TABLE 2. Pathological products and their count

\begin{tabular}{|l|c|}
\hline Pathological product & Number of samples \\
\hline Uroculture & 619 \\
\hline Sputum & 443 \\
\hline Coproculture & 149 \\
\hline Blood culture & 73 \\
\hline Wound secretion & 36 \\
\hline Bronchial aspirate & 14 \\
\hline Vaginal discharge & 9 \\
\hline Ear discharge & 7 \\
\hline Biliculture & 2 \\
\hline Pericardial fluid & 1 \\
\hline Cannula discharge & 1 \\
\hline Probe discharge & 1 \\
\hline Cerebrospinal fluid & 1 \\
\hline Pleural fluid & 1 \\
\hline Intraabdominal fluid & 1 \\
\hline
\end{tabular}




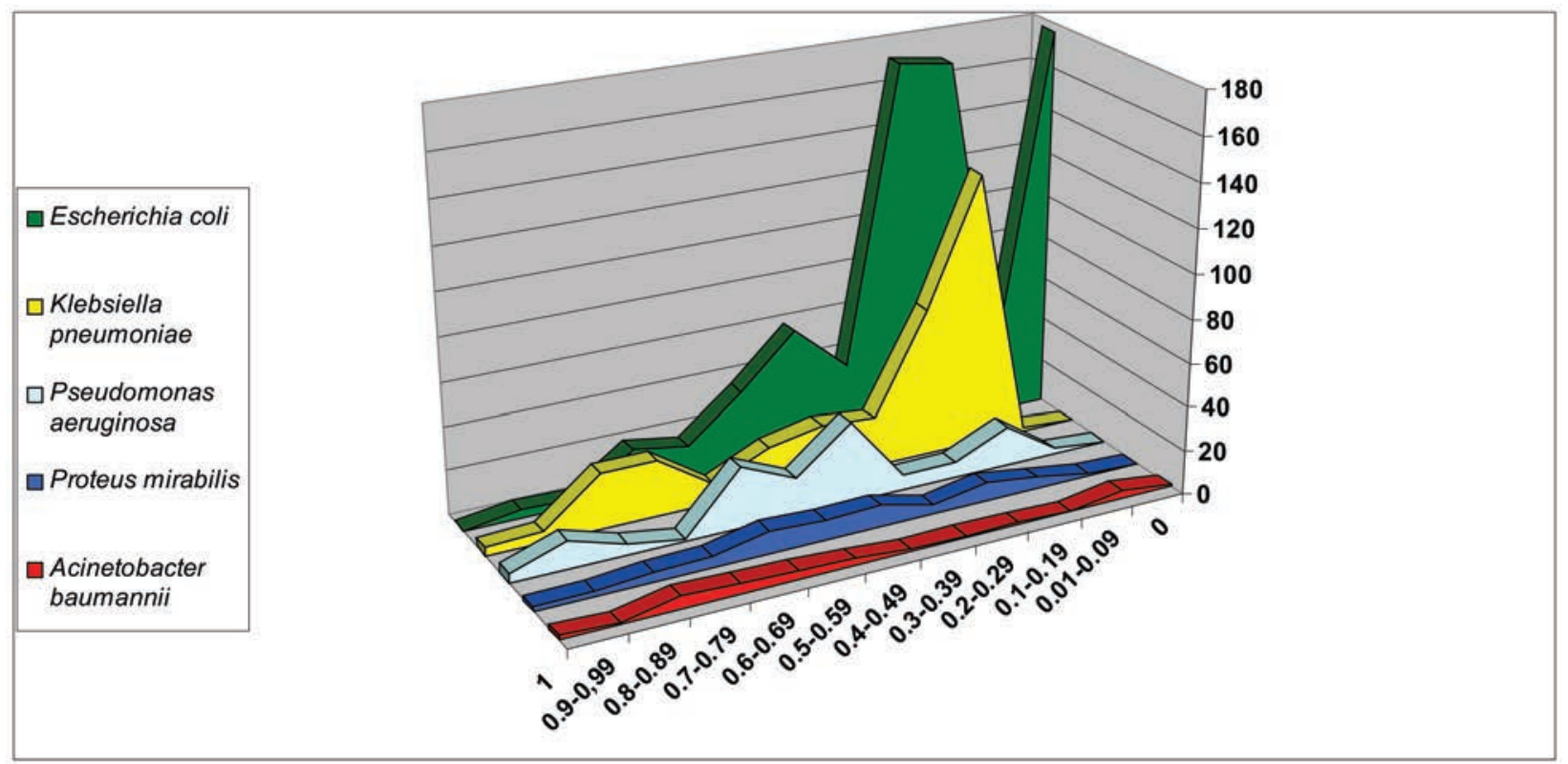

FIGURE 1. MAR index for the studied GNBS

For the first classification criteria (MAR index) the following data were obtained (mean \pm standard deviation): Ec $-0.23 \pm 0.2, \mathrm{Kp}-0.37 \pm 0.25, \mathrm{~Pa}-$ $0.51 \pm 0.24, \mathrm{Pm}-0.52 \pm 0.24, \mathrm{Ab}-0.6 \pm 0.31$. For all the strains tested, the MAR index had an average value of $0.32 \pm 0.25$. Figure 1 shows graphically the MAR index for each studied GNB strain.

For the $2^{\text {nd }}$ and $3^{\text {rd }}$ classification criteria (the percentage of MDR and XDR strains), the data are presented in Table 3.

TABLE 3. Percentage of MDR and XDR strains for the studied GNBs

\begin{tabular}{|c|c|c|c|c|c|}
\hline GNBs & Ec & Kp & Pa & Pm & Ab \\
\hline MDR (\%) & 51.94 & 68.86 & 76.67 & 58.71 & 51.72 \\
\hline XDR (\%) & 0 & 0 & 0 & 6.47 & 17.24 \\
\hline
\end{tabular}

A summative graphical situation of the antimicrobial resistance problems identified for the studied strains is presented in figure 2 .

The classification of the studied GNBs (according to their degree of antimicrobial resistance is presented in Table 4.

\section{DISCUSSION}

The issue of resistance to antimicrobials is of extremely importance to any physician. Specialists know a number of "problematic bacteria" from this point of view: some strains of Staphylococcus aureus, Enterococcus spp., Acinetobacter baumannii, Pseudomonas aeruginosa, Klebsiella spp. etc. Most of the antibiotic-resistant germs (at least according to

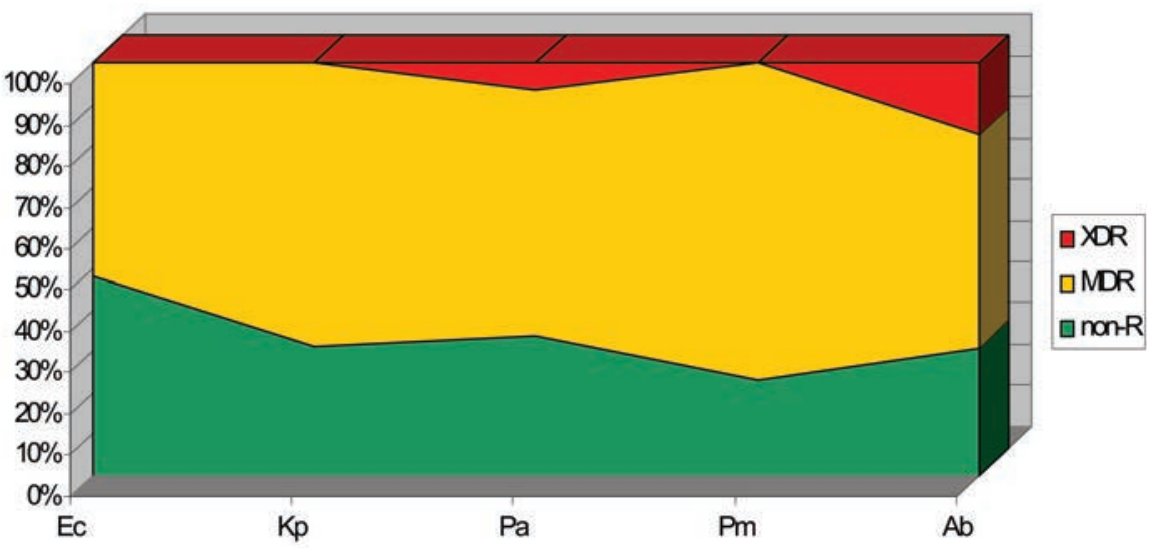

FIGURE 2. A composite graphic of the antimicrobial resistance problems for the studied GNBs 
TABLE 4. Final ranking of the studied GNBs (NA = not awarded)

\begin{tabular}{|l|c|c|c|c|c|c|c|c|c|c|}
\hline & \multicolumn{2}{|c|}{ Ec } & \multicolumn{2}{c|}{ Kp } & \multicolumn{2}{c|}{ Pa } & \multicolumn{2}{c|}{ Pm } & \multicolumn{2}{c|}{ Ab } \\
\hline Criteria & Rank & $\begin{array}{c}\text { Awarded } \\
\text { points }\end{array}$ & Rank & $\begin{array}{c}\text { Awarded } \\
\text { points }\end{array}$ & Rank & $\begin{array}{c}\text { Awarded } \\
\text { points }\end{array}$ & Rank & $\begin{array}{c}\text { Awarded } \\
\text { points }\end{array}$ & Rank & $\begin{array}{c}\text { Awarded } \\
\text { points }\end{array}$ \\
\hline MAR & 5 & 1 & 4 & 2 & 3 & 3 & 2 & 4 & 1 & 5 \\
\hline \%MDR & 4 & 2 & 2 & 4 & 1 & 5 & 3 & 3 & 5 & 1 \\
\hline \%XDR & NA & 0 & NA & 0 & 2 & 4 & NA & 0 & 1 & 5 \\
\hline Total points & & 3 & & 6 & & 12 & & 7 & & 11 \\
\hline
\end{tabular}

the WHO list) are Gram-negative bacteria. The author did not find data in the medical literature to classify these bacteria, to conclude "how much problematic" they are (at least in a certain geographical region). For the purpose of this paper an arbitrary scoring system has been used, but which, applied to each species, should not affect the final classification. One point of discussion could be the relevance of the classification related to the different number of strains isolated for each species, but the percentage expression for each classification criterion considered should be an objective point of analysis. It can also be argued whether the score should be identical

\section{REFERENCES}

1. CDC. 2020 Diseases and organisms in healthcare settings. Available at: https://www.cdc.gov/hai/organisms/organisms.html.

2. Heffner AC, Horton JM, Machick MR, Jones AE. Etiology of illness in patients with severe sepsis admitted to the hospital from the emergency department. Clin Infect Dis. 2010;50(6):814-20.

3. van der Poll T, Wiersinga WJ. Sepsis and septic shock. In: Bennett JE, Dolin R, Blasser MJ (eds.). Mandell, Douglas and Bennett's Principles and practice of infectious diseases, 9th ed. Philadelphia: Elsevier; 2020:990-1008e3.

4. WHO. 2016 United Nations high-level meeting on antimicrobial resistance. Available at: https://www.who.int/antimicrobialresistance/events/UNGA-meeting-amr-sept2016/en/.

5. WHO. 2017 WHO publishes list of bacteria for which new antibiotics are urgently needed. Available at: https://www.who.int/news-room/ for each classification criterion; at least for the data presented, it was considered that each of them has an equal share in the final classification. This study is practically an exercise and can be useful, however perfectible in the future.

\section{CONCLUSION}

The final classification of Gram-negative bacteria analyzed in terms of antimicrobial resistance problems is as follows: 1. Pseudomonas aeruginosa; 2. Acinetobacter baumanii; 3. Proteus mirabilis; 4. Klebsiella pneumoniae; 5. Escherichia coli.

Conflict of interest: none declared Financial support: none declared

detail/27-02-2017-who-publishes-list-of-bacteria-for-which-newantibiotics-are-urgently-needed.

6. Liu YY, Wang Y, Walsh TR, et al. Emergence of plasmid-mediated colistin resistance mechanism MCR-1 in animals and human beings in China: a microbiological and molecular biological study. Lancet Inf Dis. 2016;16(2):161-68.

7. Krumperman $\mathrm{PH}$. Multiple antibiotic resistance indexing of Escherichia coli to identify high-risk sources of fecal contamination of foods. Appl Environ Microbiol. 1983;46(1):165-70.

8. Magiorakos AP, Srinivasan A, Carey RB, et al. Multidrug-resistant, extensively drug-resistant and pandrug-resistant bacteria: an international expert proposal for interim standard definitions for acquired resistance, Clin Microbiol Infect. 2012;18:268-81. 